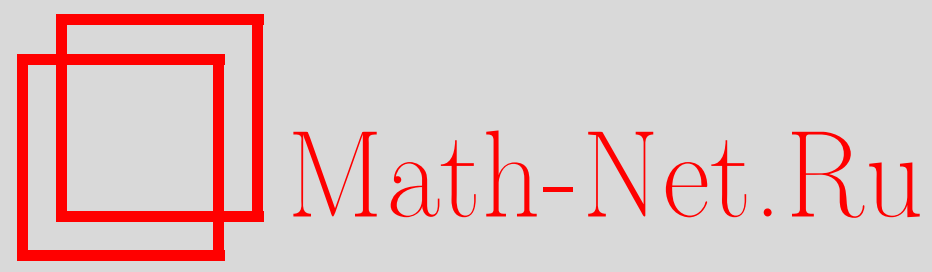

А. Ю. Жиров, Примеры одномерных гиперболических аттракторов на неориентируемых поверхностях, Матем. заметки, 1999, том 65, выпуск 3, 468-471

DOI: https://doi.org/10.4213/mzm1071

Использование Общероссийского математического портала Math-Net.Ru подразумевает, что вы прочитали и согласны с пользовательским соглашением http://www.mathnet.ru/rus/agreement

Параметры загрузки:

IP : 3.93.64.190

26 апреля 2023 г., 08:01:10

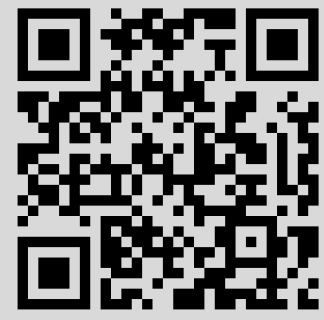




\section{ПРИМЕРЫ ОДНОМЕРНЫХ ГИПЕРБОЛИЧЕСКИХ АТТРАКТОРОВ НА НЕОРИЕНТИРУЕМЫХ ПОВЕРХНОСТЯХ}

\section{А. Ю. Жиров}

В настоящей работе рассматриваются некоторые вопросы существования диффеоморфизмов неориентируемых поверхностей с гиперболическими аттракторами. Под аттракторомпонимается компактное связное подмножество $\Lambda$ замкнутой компактной поверхности $M$, инвариантное и гиперболическое относительно диффеоморфизма $f: M \rightarrow M$, причем такое, что существует его окрестность $U$ в $M$, для которой $f(U) \subset U$ и $\bigcap_{n \geqslant 0} f^{n}(U)=\Lambda$. Аттракторы естественно рассматривать с точностью до топологической сопряженности порождающих их диффеоморфизмов на некоторых их окрестностях. В этом смысле говорят об окрестностной сопряэсенности аттракторов. С ее учетом будем понимать реализуемость аттрактора на поверхности. Именно, рассмотрим следу-

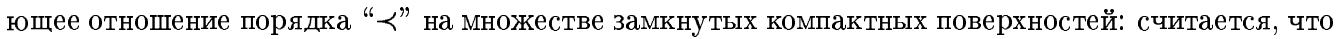
$M_{1} \prec M_{2}$, если либо $M_{1}$ ориентируема, а $M_{2}$ неориентируема, либо обе они ориентируемы или обе неориентируемы, а род $M_{1}$ меньше рода $M_{2}$. Тогда $M$ называется минимальной поверхностью аттрактора $\Lambda$ диффеоморфизма $f: M \rightarrow M$, если не существует окрестностно сопряженного ему аттрактора на поверхности $M^{\prime} \prec M$. Говоря об аттракторе на данной поверхности, будем иметь в виду, что она является его минимальной поверхностью.

Напомним некоторые геометрические свойства аттракторов, связанные с топологическими характеристиками его минимальной поверхности (см. [1], [2]). Для каждой точки $x \in \Lambda$ устойчивое $W^{s}(x)$ и неустойчивое $W^{u}(x)$ многообразия суть образы $\mathbb{R}$ при инъективньх иммерсиях в $M$, причем $\Lambda$ является объединением неустойчивых многообразий своих точек. Точка $y \in \Lambda$ назьвается оппозиционной для точки $x \in \Lambda$ тогда и только тогда, когда $y \in W^{s}(x)$ и $(x, y)_{s} \cap \Lambda=\varnothing$. Подмножество достижимой извне границы аттрактора называется связкой, если вместе с любой его точкой $x$ в нем содержится все множество $W^{u}(x)$, а также точка, оппозиционная к $x$ (если такая существует), и если оно не содержит собственного подмножества с указанными свойствами. Достижимая граница аттрактора распадается в дизъюнктное объединение конечного числа связок, причем каждая из них есть дизъюнктное объединение конечного числа неустойчивых многообразий точек аттрактора. Число неустойчивых многообразий, составляющих связку, называется ее степенью. Последовательность чисел $\left\{b_{d}: d \in \mathbb{N}\right\}$, где $b_{d}$ есть число связок степени $d$, называется граничньм типом аттрактора. Он связан с эйлеровой характеристикой минимальной поверхности аттрактора формулой Эйлера-Пуанкаре

$$
b_{1}-\sum_{d \geqslant 3}(d-2) b_{d}=2 \chi(M) .
$$

Кроме граничного типа аттрактора его реализуемость на поверхностях зависит еще от одного свойства, назьваемого ориентируемостью. Оно означает ориентируемость ламинации, порождаемой разбиением аттрактора на неустойчивые многообразия его точек (см. [1]).

Возникает вопрос о том, какие граничные типы ориентируемых и неориентируемых аттракторов, формально допускаемые формулой Эйлера-Пуанкаре для данной поверхности, в действительности реализуются. В случае ориентируемых поверхностей ответ известен благодаря результату [3] (см. также [2]), а в случае неориентируемых остается открытым. Известно только, что на бутылке Кляйна не существует аттракторов без 1-связок [4]. В [5] построен пример обобщенного псевдоаносовского гомеоморфизма проективной плоскости, из которого в соответствии с конструкцией $[6]$ получается диффеоморфизм с аттрактором граничного типа $\left\{b_{1}=3, b_{3}=1\right\}$. В настоящей работе методами [2] приводятся примеры диффеоморфизмов с аттракторами, доказывающие следующие утверждения.

Работа выполнена при финансовой поддержке Российского фонда фундаментальных исследований, грант № 97-01-00301, совместного проекта РФФИ и фонда INTAS, грант № 96-00418i96, и совместного проекта РФФИ и фонда ННИО, грант № 96-01-00043G. 
Теорема 1. Для любого $d \geqslant 3$ на проективной плоскости существует аттрактор граничного типа $\left\{b_{1}=d, b_{d}=1\right\}$.

ТЕОРема 2. Существуют аттракторы диффеоморфизмов проективной плоскости, несопряжсенные аттракторам диффеоморфизмов ориентируемых поверхностей и имеющ,ие сколь угодно малую топологическую энтропию.

ТЕОРема 3. На неориентируемой поверхности рода 5 существуют ориентируемые аттракторы всех граничных типов, допускаемых в әтом случае формулой ЭйлераПуанкаре. Без учета аттракторов с 2-связками эти граничные типы суть $\left\{b_{8}=1\right\}$, $\left\{b_{4}=b_{6}=1\right\} u\left\{b_{4}=3\right\}$.

ТЕОРема 4. На кахдой неориентируемой поверхности четного рода $g \geqslant 4$ существует $A$-диффеоморфизм $f_{g}$, спектральное разложение которого состоит из единственной отталкивающей неподвижной точки и ориентируемого аттрактора граничного типа $\left\{b_{2 g-2}=1\right\}$. При этом топологическая энтропия диффеоморфизма $f_{g}$ есть логарифм максимального корня полинома $\lambda^{g-1}-\lambda^{g-2}-\lambda-1$, и поэтому $\operatorname{ent}\left(f_{g}\right) \rightarrow 0$ nри $g \rightarrow \infty$.

Построение примеров, доказьвающих эти теоремы, основано на комбинаторном описании аттракторов, данном в [2] для случая диффеоморфизмов ориентируемых поверхностей. Коротко повторим эту конструкцию с дополнениями, необходимыми для случая неориентируемых поверхностей.

Для аттрактора $\Lambda$ диффеоморфизма $f: M \rightarrow M$ строится двумерное подмногообразие $\Pi \subset M$ с краем, называемое фундаментальным многообразием аттрактора. Оно обладает так называемым ленточным разбиением [7], т.е. получается в результате приклеивания к дуге $I$, называемой базовым отрезком, некоторого количества (обозначим его через $n$ ) евклидовых прямоугольников $\Pi_{i}$, называемых лентами, вдоль пар противоположных сторон. Точные определения сформулированы в [7], где доказывается существование фундаментальных многообразий для любого аттрактора (для этого, правда, приходится сделать ненужное сейчас обобщение, рассматривая ленточные поверхности с, вообще говоря, несколькими базовьми отрезками). Диффеоморфизм, порождающий аттрактор, отображает П на свое подмножество, причем это отображение согласо-

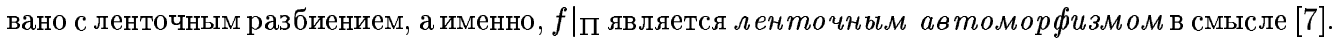
Эти свойства позволяют дать следующее комбинаторное описание фундаментального многообразия и отображения $\left.f\right|_{\Pi}$.

Выберем определенную ориентацию базового отрезка (скажем: слева направо) и определенное трансверсальное направление к нему (скажем: снизу вверх). Стандартным образом занумеруем отрезки, вдоль которых ленты приклеены к $I$, перечислив сначала приклеенные к $I$ сверху, а затем - приклеенные снизу в соответствии с направлением слева направо. Возникает подстановка $\sigma$ на множестве $1, \ldots, 2 n$, сопоставляющая друг другу номера отрезков, вдоль которых приклеена к базовому отрезку одна и та же лента. Далее занумеруем сами ленты $\Pi_{i}$, согласуя их нумерацию с нумерацией отрезков, к которым они приклеены, и сопоставим номеру каждой из них знак "+" или “-", указьвающий на то "перекручена" она при приклеивании или нет. Получим $\boldsymbol{n}$-вектор $\boldsymbol{o}$, компонентами которого являются знаки. Наконец, определим число $t$ отрезков, вдоль которых ленты приклеены к $I$ сверху. Набор данных $\mathrm{cf}:=(\sigma, t, \boldsymbol{o})$ называется конфигурачией ленточной поверхности.

Отображению $f: \Pi \rightarrow \Pi$ сопоставляется следующий набор данных $\left\langle\mathrm{cf} ; \varepsilon^{u}, \varepsilon^{s} ; \boldsymbol{g}, l\right\rangle$, назьваемый кодом аттрактора:

cf есть конфигурация ленточной поверхности П;

$\varepsilon^{s}$ (соответственно $\varepsilon^{u}$ ) есть знак "+" или "-" в зависимости от того, сохраняет $f$ ориентацию отрезка $I$ (соответственно трансверсальное направления к нему) или нет;

$\boldsymbol{g}=\left(g_{1}, g_{2}, \ldots, g_{n}\right)$ есть целочисленный $n$-вектор с компонентами $g_{i}:=\#\left(\Pi_{i} \backslash I\right) \cap f(\Pi)$, где “\#” означает число связных компонент множества;

наконец, $l:=\# L I \cap f(\Pi)$, где $L I$ - левый отрезок (возможно пустой и тогда $l:=0$ ) из тех двух, которые получаются, если из $I$ удалить $f(I)$.

Так как в [2] рассмотрен случай аттракторов диффеоморфизмов ориентируемых поверхностей, там фигурируют коды с $\boldsymbol{o}=(+\cdots+)$, и, кроме того, с $\varepsilon^{s}=+$. Установлены необходимые и достаточные условия того, что формально записанный код такого типа действительно реализуется 
некоторым диффеоморфизмом поверхности с аттрактором. Их нетрудно распространить и на коды описанного вьше типа (и даже более общего), на основании чего можно убедиться в реализуемости формальных кодов, приведенных в следующих ниже примерах.

ПРИмеР 1. Пусть $n \geqslant 4, t:=4$,

$$
\sigma:=(1,2 n)(2,5)(3,4) \prod_{i=3}^{n-1}(2 i, 2 i+1), \quad \boldsymbol{o}:=(--\underbrace{+\cdots+}_{n-2}) .
$$

Для конфигурации $(\sigma, t, \boldsymbol{o})$ определим формальный код, полагая $\varepsilon^{u}=\varepsilon^{s}=+, l=0$ и

$$
\boldsymbol{g}:=(5,3,3, \underbrace{1, \ldots, 1}_{n-4}, 3) .
$$

Этот код определяет аттрактор на проективной плоскости граничного типа $\left\{b_{1}=n-1, b_{n-1}=1\right\}$, что доказывает теорему 1.

ПРИмеР 2 . Пусть $k \geqslant 1, n:=2 k+2, t:=2 k+1$,

$\sigma:=(1,2 n-1) \prod_{i=1}^{k}(2 i, 2 i+1) \cdot(2 k+2,2 n) \cdot \prod_{i=k+1}^{2 k}(2 i+1,2 i+2), \quad \boldsymbol{o}:=(-\underbrace{+\cdots+}_{k}-\underbrace{+\cdots+}_{k})$.

Для этой конфигурации определим формальный код, полагая $\varepsilon^{u}=-, \varepsilon^{s}=+, l=0$,

$$
\boldsymbol{g}:=(3, \underbrace{1, \ldots, 1}_{k-1}, 3, \underbrace{1, \ldots, 1}_{k+1}) .
$$

Он определяет аттрактор граничного типа $\left\{b_{1}=2 k+1, b_{2 k+1}=1\right\}$ на проективной плоскости, причем оказывается, что топологическая энтропия последнего есть логарифм максимального корня полинома $\lambda^{2 k+1}-\lambda^{2 k}-\lambda-1$, а этот корень, как нетрудно проверить, стремится к 1 при $k \rightarrow \infty$. Это доказьвает теорему 2 .

ПримеР 3. Пусть $k \geqslant 2, n:=2 k, t:=k$. Положим

$$
\sigma:=(1,3 k)(k+1,2 n) \prod_{\substack{i \geqslant 2 \\ i \neq k+1}}(i, 2 n-i+1), \quad \boldsymbol{o}:=(-\underbrace{+\cdots+}_{k-1}-\underbrace{+\cdots+}_{k-1}) .
$$

Для конфигурации $(\sigma, t, \boldsymbol{o})$ определим формальный код, полагая $\varepsilon^{u}=-, \varepsilon^{s}=+, l=2$,

$$
\boldsymbol{g}:=(2, \underbrace{1, \ldots, 1}_{k-1}, 2, \underbrace{1, \ldots, 1}_{k-2}, 3) .
$$

Он определяет аттрактор граничного типа $\left\{b_{2 n-2}=1\right\}$ на неориентируемой поверхности рода $g=n$ со свойствами, указанными в теореме 4 .

Наконец, теорему 3 доказывают следуюшие три примера кодов, которые определяют на неориентируемой поверхности рода 5 ориентируемые аттракторы граничных типов, указанных (в той же последовательности) в этой теореме:

1) $\sigma=(1,9)(2,6)(3,7)(4,10)(5,8), \quad t=5, \quad \boldsymbol{o}=(+-++-)$, $\varepsilon^{u}=+, \quad \varepsilon^{s}=-, \quad g=(4,2,3,4,6), \quad l=11$;

2) $\sigma=(1,11)(2,9)(3,7)(4,8)(5,12)(6,10), \quad t=6, \quad \boldsymbol{o}=(++-++-)$, $\varepsilon^{u}=+, \quad \varepsilon^{s}=+, \quad g=(7,2,3,5,1,3), \quad l=3$;

3) $\sigma=(1,13)(2,11)(3,8)(4,10)(5,14)(6,9)(7,12), \quad t=7, \quad \boldsymbol{o}=(++-+++-)$, $\varepsilon^{u}=-, \quad \varepsilon^{s}=-, \quad g=(5,1,4,1,2,2,4), \quad l=5$. 


\section{СПИСОК ЦИТИРОВАННОЙ ЛИТЕРАТУРЫ}

1. Плыкин Р. В. // УМН. 1984. Т. 39. №6. С. 75-113. 2. Жииров А. Ю. // Матем. сб. 1994. Т. 185. №6. С. 3-50; // Матем. сб. 1994. Т. 185. №9. С. 29-80. 3. Mazur H., Smillie J. // Comment. Math. Helv. 1993. V. 68. P. 289-307. 4. Плыкин Р. В. // УМН. 1980. Т. 35. № 4. C. 205-206. 5. Arnoux P., Yoccoz J.-P. // C. R. Acad. Sci. Paris. Sér. I. Math. 1981. V. 292. Р. 75-78. 6. ЖКиров А. Ю., Плыкин Р. В. // Матем. заметки. 1995. Т. 58. №1. С. 149-152. 7. ЖКиров А. Ю. // Матем. сб. 1997. Т. 188. №6. С. 3-26. 